\title{
In Search of Family Resilience
}

\author{
Weining C. Chang1, Anna H. C. Neo ${ }^{2}$, Daniel Fung ${ }^{3}$ \\ ${ }^{1}$ Graduate Medical School, Duke-NUS, Singapore City, Singapore \\ ${ }^{2}$ Nanyang Technological University, Singapore City, Singapore \\ ${ }^{3}$ Institute of Mental Health, Singapore City, Singapore \\ Email: weiningchu.chang@duke-nus.edu.sg
}

Received 19 August 2015; accepted 6 October 2015; published 9 October 2015

Copyright (C) 2015 by authors and Scientific Research Publishing Inc.

This work is licensed under the Creative Commons Attribution International License (CC BY). http://creativecommons.org/licenses/by/4.0/

c) (i) Open Access

\section{Abstract}

This study explores the construct of family resilience defined as a family variable that enables the family to survive major life challenges and as a coping resource that enables the individual to deal with traumas and to thrive from the experience. Nurses who were working with infected patients in a major epidemic in Singapore provided the sample and the context to search for family resilience. Two studies are reported in this article: Study 1 consisted of an in-depth interview with 30 nurses and some of their family members to identify the factors within their families that they perceived to have enabled them to cope with the stress and difficulties. Based on the results from Study 1, a family resilience (FR) scale was constructed. Using the FR scale, Study 2 employed a structured survey to identify the internal organization and the psychometric properties of FR. Factor analysis of the results identified five meaningful factors. The FR scale was found to have excellent psychometric properties. Path analyses were conducted to test whether family resilience is a spurious factor and whether it is a construct distinct and independent from individual resilience (IR). It was found that FR predicts individual well-being with both direct and indirect effect mediated by individual resilience, supporting the nonspurious nature of FR and the potential reciprocal causal effects between FR and IR.

\section{Keywords}

Family Resilience, Individual Resilience, Stress and Coping, Subjective Well-Being

\section{Introduction}

In several major epidemics and natural disasters that affected numerous Asian countries, Singapore was exemplary in its efficient management and effective coping responses used to contain the damage (Chang \& Sivam, 2004). In the aftermath of the crises, many medical and paramedical professionals risked their own lives 
to work with victims and families. In times of such crises, nurses and emergency doctors often had to be quarantined or at least separated from their families (Masten \& Obradovic, 2007). The stress induced by an uncontrollable, devastating event often takes a great toll on the family (for example, McCubbin, 1982; Patterson, 1988, 2002).

Crises encountered by the family frequently cause friction and even disintegration of the family process and structure. However, in our earlier study on coping with an epidemic in Singapore (Chang \& Sivam, 2004), we came across many incidences where the participants voluntarily mentioned the support of their families. From their reports, a pattern of Singaporeans coping with uncontrollable traumas and natural disasters emerged; that is, they coped as families and not as isolated individuals, and the family functioned as a unit of response. This led us to the hypothesis that in this collectivistic Asian country (Hofstede, 1980), where people come from a long history of having family-oriented cultures (Bentelspacher, Chitran, \& Rahman, 1994), individuals, when faced with major life threats, cope in a collectivistic way, whereby the family functions as a team or an integrated unit in the stress-and-coping process. The construct of family resilience (Walsh, 1998a, 2003; Patterson, 2002) was introduced as a family characteristic that enables families to withstand and rebound from the disruptive challenges they face.

We chose to focus on the family as a functional unit for coping with adversity and applied the stress-andcoping framework (Lazarus \& Folkman, 1984) to identify a set of familial factors and processes that provide the family and the individual with protective effects (Cohen, Slonim, Finzi, \& Leichtentritt, 2002; Lobo, 2008; De Haan, Hawley, \& Deal, 2002). In other words, we searched for characteristics and processes in Singaporean families that constitute family resilience (FR).

Resilience is defined (Carver, 1998; Rutter, 1987, 1999) as a psychological construct that moderates the stress-and-coping process and contributes to the coping outcomes by reducing negative effects and enhancing positive outcomes (Bonanno, Ho., Chan, Kwong, Chueng, \& Wong, 2008; Fletcher \& Sarkar, 2013; McCrae \& Costa, 1993). Individual psychological resilience has been defined as the underlying process or trait that intervenes in the person-situation transaction that empowers the individual to survive and thrive in the face of adversity (Bonanno, 2004; Garmezy, 1985; Luthar, 2006).

Psychological resilience, although identified as an individual-level variable (Carver, 1998; Chang \& Lim, 2007; Rutter, 1987), could be applied to organizations or social systems when challenged by trauma and disaster (Marsten \& Obradovic, 2008; Patterson, 2002; Rutter, 1999). At the system level, family resilience denotes a set of dynamic factors in the family process that enable the individual family member to weather life's challenges (Patterson, 2002; Walsh, 1998a, 2003). In line with current literature on family resilience, we propose that the family, as a social system and the unit of function in most Asian communities, can be treated as an agent or an actor in the stress-and-coping process (for example, McCubbin, 1982; Patterson, 1988, 2002). Likewise, Masten \& Obradovic (2007) observe that families should be treated as a response unit in times of natural disaster, war, pandemics such as SARS, and terrorism.

In their seminal work on family resilience, Walsh (1998a, 2003) and Patterson (2002) identify a number of key factors that constitute family resilience, namely a shared family belief system, a flexible family structure, and effective communication. Under these broad categories, specific manifestations and operations characterize family members' interactions with each other that, in turn, lead to effective coping when faced with major life challenges. Analyzing the ecological context of human development, Bronfenbrenner (1986) observes that family, like the individual, is an embedded entity that would be influenced by larger social-ecological contexts. Family resilience, therefore, would be conceptualized and operationalized within a larger sociocultural context. Likewise, scholars who study families in different cultures also found that family resilience varies as a function of cultural values and beliefs (Patterson, 2002; Walsh, 1998b). In the present study, instead of using the categories identified in Western literature, we conducted a series of empirical studies with the aim of identifying the local conceptualization of family resilience and its specific manifestations.

We embarked on the search for family resilience by conducting ethnographic interviews with nurses who worked with SARS patients and the families of these nurses. We aimed to determine from their experiences what enabled them to effectively cope with the threat of SARS and deal with the risks. These qualitative results were coded for major categories of responses and recurring themes to identify characteristics of the family that shielded the nurses from the negative effects of anxiety and depression while enhancing their subjective well-being. 
By using a combination of qualitative and quantitative methods, firsthand data were obtained to identify the indigenous conceptualization of family resilience (Cohen et al., 2002; Lee, Lee, Kim, Park, Song, \& Park, 2004).

\section{Study 1}

\subsection{Conceptualization and Manifestation of Family Resilience}

The identification of psychological resilience in the traditional literature on human development has been in the context of life adversity (for instance, Garmecz, 1985). In the recent history of Asia, psychological resilience was often observed in the context of catastrophes caused by uncontrollable natural phenomena (Chang, 2007).

The following are some direct quotes from the nurses who worked with SARS patients in several Singaporean hospitals: “This is too big for me to deal with!” “This is something beyond me.” "I need my family’s support to deal with it!" “Thank God my family is behind me, otherwise...” "I consult my family to gain a better understanding of what happened."

As can be inferred from these quotes, when the nurses were asked to work with SARS patients, they turned to their families for the strength to deal with the challenge of working under high-risk conditions. They drew resilience from their families as a reliable coping resource.

We constructed a semistructured questionnaire to interview the nurses on issues concerning how and why they coped with these experiences, to establish whether they coped well, and to determine what helped them to cope well. We specifically asked what happened within the family that enabled them to cope well.

\subsection{Method}

\subsubsection{Participants}

Fifteen Singaporean Chinese nurses (one male and 14 females) and 15 Singaporean Malay nurses (two males and 13 females), with ages ranging from 30 to 56 and more than 10 years of seniority in the nursing profession, from one of the national hospitals where some of the patients and staff were suspected of being infected and quarantined during the pandemic outbreak were recruited to participate in the interviews. Two of the Chinese nurses' families (spouses and children), two of the Malay nurses' families (spouses and children), and one Malay nurse's mother were also recruited. Among the nurses, 27 were female and three were male. Eight of the nurses worked in the isolation ward for SARS patients during the SARS outbreak.

\subsubsection{Measures}

Semistructured interview questions were used to elicit responses from the nurses and their family members. The interviews consisted of a set of open-ended English questions, tapping the participants' experiences of how they coped with stress and practical inconvenience brought by having to work with SARS patients during the outbreak. The interview sought to identify major sources and factors that provide resilience as perceived by these nurses and their families.

\subsubsection{Procedures}

The nurses (15 Chinese nurses and 15 Malay nurses) were recruited from one national hospital, with two families of the Chinese nurses and two families of the Malay nurses also participating in this study. The semistructured in-depth interviews were conducted with these nurses at their working ward, and the family members were interviewed at their homes. The average length of each interview was two hours. The interviews were conducted in English, with some common Malay expressions to clarify important concepts to the Malay nurses. The interviewers included one Chinese and one Malay psychology major students from a local university. The two interviewers were bilingual, speaking English and their mother tongues-Malay or Chinese. The interviews were recorded on audio devices, and, where the participants granted permission to the interviewers, the interviews were also recorded on a video recorder.

The participants' audio-recorded responses were transcribed into text scripts. Two experienced psychologists identified recurring themes from the scripts. These themes formed the categories of responses, which were sys- 
tematically coded for their frequencies in each category by two independent raters. The two raters reached $80 \%$ agreement in the coding of responses. Final coding results were obtained by consensus discussions between the raters. Finally, a questionnaire measuring family resilience was constructed using the most frequent responses from the interview data.

\subsection{Results}

Recurring themes identified from participants' responses were used to form categories of responses that define family resilience. Participants' responses were coded in frequencies of occurrences, according to the response categories. There were 239 responses from the Chinese participants and 280 responses from the Malay participants. The frequencies of responses have been summarized in Table 1 and Table 2 for the Malay and Chinese participants, respectively.

The two ethnic groups showed similar resilience factors, scoring high frequencies in areas involving the family as a unit, such as "cooperation" (Malay 13.2\%, Chinese 13\%) and "being united" (Malay 10\%, Chinese 20.1\%). Interaction and information sharing (that is, communication) within the family also appeared high in the ranking for both groups, with the Malay nurses scoring $16.8 \%$ and the Chinese nurses scoring 18.8\%. In addition, both Malay and Chinese nurses showed a preference to engage practical attitudes and strategies in dealing with stressful situations during the SARS outbreak. Coping by means of making sense of the situation ("redefine meaning of difficulties"; $2.9 \%$ in Chinese nurses) or simply accepting the situation (Malay nurses 2.1\%, Chinese nurses 7.1\%) and trying to live life as usual ("normalization," 2.9\% respectively in both Malay and Chinese nurses) were among the lower-ranked factors that have helped both Malay and Chinese families to be resilient.

However, differences between the groups were apparent when Malay families coped by involving the family in routine "religious practices" (7.9\%), while Chinese families coped by looking up to the "pillar in the family" (7.5\%) to lead them in times of crisis. Although the two ethnic groups showed differences in specific family resilience resources, they reflected family values and belief systems as sources of resilience (Walsh, 1998b). Chinese families also uniquely exhibited a strong inclination toward control and suppression of negative emotions

\begin{tabular}{lcc}
\hline \multicolumn{1}{c}{ Table 1. Frequencies and ranking for Malay resilience responses. } & \\
\hline \multicolumn{1}{|c}{ Frequencies and Ranking for Malay Resilience Responses } & Rank \\
\hline \multicolumn{1}{|c}{ Malay Categories } & Frequency \% & 1 \\
\hline Communication & 16.8 & 2 \\
Cooperation & 13.2 & 3 \\
Family love and support & 10.7 & 4 \\
Being united & 10 & 5 \\
Practicality (do what you can) & 8.6 & 6 \\
Religious practices & 7.9 & 7 \\
Positive attitude & 6.8 & 8 \\
Have a pillar in the family & 5 & 9 \\
Sacrtifice and accommodate & 4.6 & 10 \\
Prepared (for what might come) & 3.9 & 10 \\
Religious beliefs & 3.6 & 11 \\
Create lighter mood & 3.6 & 12 \\
Normalization (live as normal) & 2.9 & 13 \\
Acceptance & 2.1 & \\
\hline
\end{tabular}


Table 2. Frequencies and ranking for Chinese resilience responses.

\begin{tabular}{lcc}
\hline \multicolumn{1}{c}{ Frequencies and Ranking for Chinese Resilience Responses } & \\
\hline \multicolumn{1}{c}{ Chinese Categories } & Frequency \% & Rank \\
\hline Being united & 20.1 & 1 \\
Communication & 18.8 & 2 \\
Cooperation & 13 & 3 \\
Have a pillar in the family & 7.5 & 4 \\
Control negative emotions & 7.5 & 4 \\
Practicality & 7.5 & 4 \\
Acceptance & 7.1 & 5 \\
Religious practices & 5 & 6 \\
Religious beliefs & 3.8 & 7 \\
Family interdependence & 3.8 & 7 \\
Normalization & 2.9 & 8 \\
Redefining meaning of difficulties & 2.9 & 8 \\
\hline
\end{tabular}

(7.5\%), which contrasted with Malay families' relatively proactive attempts to "create lighter mood" (3.6\%) in the family.

In summary, both cultures exhibited their strength by uniting the family in regular interactions and engaging a practical approach to what can be done to deal with the stressful situation brought about by the SARS outbreak. Both the Malay and Chinese families in the study identified communication as a key factor in family resilience for effective coping during the period the nurses were working with SARS patients. Interestingly, spirituality has been reported by the participants as an important resilience factor, which is consistent with the findings of Western studies on family resilience (for instance, Patterson, 2002; Walsh, 1998b). Malay nurses and Christian nurses attributed their resilience to religiosity, whereas non-Christian Chinese nurses identified ancestors as their spiritual source, reflecting differences in the participants' cultural origins.

\subsection{Discussion}

In the present study, we found that participants identified a number of recurring themes as constituting resilience. They are 1) process variables: effective communication, altruistic sacrificing for the family, and accommodating the family, and loving and caring for each other; 2) structural variables: having strong leadership, being united, and being interdependent with each other; 3) collective emotional management: normalization of mood, creating a lighter mood, and controlling negative feelings; and 4) meaning making through shared belief systems, be it religion or worldviews.

Compared with the factors of family resilience reported in the West (Walsh, 1998a; Patterson, 2002), family structure factors differ in emphasis. In the West, flexibility (especially where power distribution or division of labor in the family is concerned) is considered a key resilience factor (Walsh, 2003), but in Singapore, interdependence, where each individual does her part under strong leadership, has been identified as an important resilience factor. In Singapore, accommodating and making altruistic sacrifices are considered resilient, whereas in the West, the respect of each individual's boundaries is considered resilient. Consistent with contemporary literature in individual resilience, in this Asian sample, finding meaning in life's challenges is an important family resilience factor. Therefore, both Eastern and Western families have identified making sense of the crisis (Patterson, 2002) within the framework of shared meanings of the family as an important resilience factor. Hope and optimism, the contemporary pillars of positive psychology (Seligman \& Cszikszentmihalyi, 2000), have found their way into the concept of family resilience. Hope and optimism in this context, however, are cited as ways of collectively managing the negative feelings caused by the challenge of SARS. The identification of a positive 
outlook and the normalization of a situation have been found in both Singaporean and Western studies as ways of providing resilience.

Therefore, with the exception of family structural factors, there are more similarities than differences between the factors identified in Singapore and those identified in Western literature (for instance, Stinnett \& De Frain, 1985; Walsh, 1998a, 2003). The importance of a positive outlook and positive emotions mentioned by these families also echoed the findings in individual resilience studies (Rutter, 1987, 1999).

We further refined the items to reduce overlaps and created the family resilience scale to test the family resilience construct in a larger sample in Study 2.

\section{Study 2}

Study 2 was conducted to 1 ) test the validity of the family resilience construct and its operationalization, and 2) its psychometric properties. More importantly, we wished 3) to establish that the construct of family resilience, as a moderating factor in stress and coping, is not a spurious construct of individual resilience. As a viable and independent construct, it should contribute independently to the adaptive outcomes. As a factor of resilience, it should reduce negative outcomes and enhance positive outcomes in the stress-and-coping process.

In the present study, experience with SARS was the stressor. We tested the resilient effect of the family resilience construct within this context. The Asian well-being (ASWB) scale was chosen as the positive outcome measure.

\subsection{Method}

\subsubsection{Participants}

A total of 188 participants, consisting of 111 nurses and 77 family members of the nurses, participated in the study. Among them, 127 were female and 56 were male, and five participants did not indicate their gender identification. The nurses were recruited from two major Singaporean hospitals that admitted SARS patients and patients suspected of being infected with SARS during the outbreak. The family members were parents, spouses, and/or children of the nurses. The mean age of the nurses was 38.43 years, while the mean age of the family members was 36.91 years. There were 118 Chinese, 29 Malay, 29 Indian, and 12 unidentified participants. Eighty-one of them were protestant or Catholic Christians, 31 were Buddhists or Taoists, 34 were Muslims, 15 were Hindus, 25 were freethinkers, and two did not indicate any religious beliefs.

\subsubsection{Measures}

Three English/Chinese paper-and-pencil questionnaires were administered (see Appendix). These questionnaires were 1) family resilience scale, 2) individual resilience scale (Chang \& Lim, 2007), and 3) abbreviated 15-item Asian well-being scale (Chu, 2002). The first scale, the family resilience scale, was especially constructed for the present study to answer the main research question. The 21-item scale was constructed based on the qualitative interview data obtained from Study 1. The following section provides detailed descriptions of each of the questionnaires used:

The family resilience scale was constructed from the most frequent or recurring themes identified in Study 1. It consists of 21 items that assess how families deal with adversity, as perceived by the individual (for example, "We control negative emotions," and "We accommodate one another's needs"). Respondents were required to indicate to what extent they agreed or disagreed with each statement, using a 5-point Likert scale, ranging from 1 (strongly disagree) to 5 (strongly agree). A higher score would indicate a higher level of resilience in the family as perceived by the individual. The reliability alpha obtained for the present study is 0.90 .

The individual resilience scale (Chang \& Lim, 2007) is a locally constructed scale that measures the belief systems and practices that contribute to the resilience or positive adaptation of individuals in the face of adverse conditions. It consists of three dimensions: a) implicit beliefs of self, b) emotional self-regulation, and c) coping flexibility, with a total of 27 items (6 items, 9 items, and 12 items, respectively). Cronbach's alpha for the overall scale used in the present study was 0.88 .

The Asian well-being scale is an abbreviated 15-item version of the initial 30-item scale (Chu, 2002), obtained by performing split-half reliability (alpha $=0.90$ ) on the original scale. The Asian well-being scale meas- 
ures how an individual feels about his or her life (for example, "I have a balanced life"). Respondents were required to indicate the extent to which each statement described their current state on a 5-point scale, ranging from "doesn't describe me at all” to "describes me very much.” The initial 30-item scale reportedly has alphas of 0.92 and 0.81 for the individual well-being factor and the collective well-being factor, respectively. The 15-item version used in the current study transformed the initial short-phrase items into full-sentence items. The reliability alpha obtained for the present study was 0.93 .

\subsubsection{Procedures}

The participants were nurses recruited from two hospitals that treated SARS patients in Singapore. The questionnaires were sent to the nurses through the nursing administration department in the respective hospitals. Each participant was given two sets of the same questionnaires. The nurses were required to fill out one set of the questionnaires by themselves and to ask one of their family members to fill out the other set. The nurses were informed that they could contact us for more questionnaires should more than one of their family members like to participate in the study. Upon completing the questionnaires, they were requested to return them to us in sealed envelopes through the nursing administration departments of their hospitals.

\subsection{Results}

\subsubsection{Factor Analysis (1): Structure of Family Resilience}

Exploratory factor analysis was conducted on 21 items of the family resilience scale to determine the underlying dimensions of family resilience. The analysis was based on the responses from 179 participants after deleting nine multivariate outliers. Five components with eigenvalues greater than one were extracted using principal component analysis. The scree test confirmed the selection of a five-factor solution (see Figure 1). Hence, principal component analysis via varimax rotation was performed to obtain a more interpretable five-factor solution. The loadings of the items are presented in Table 3.

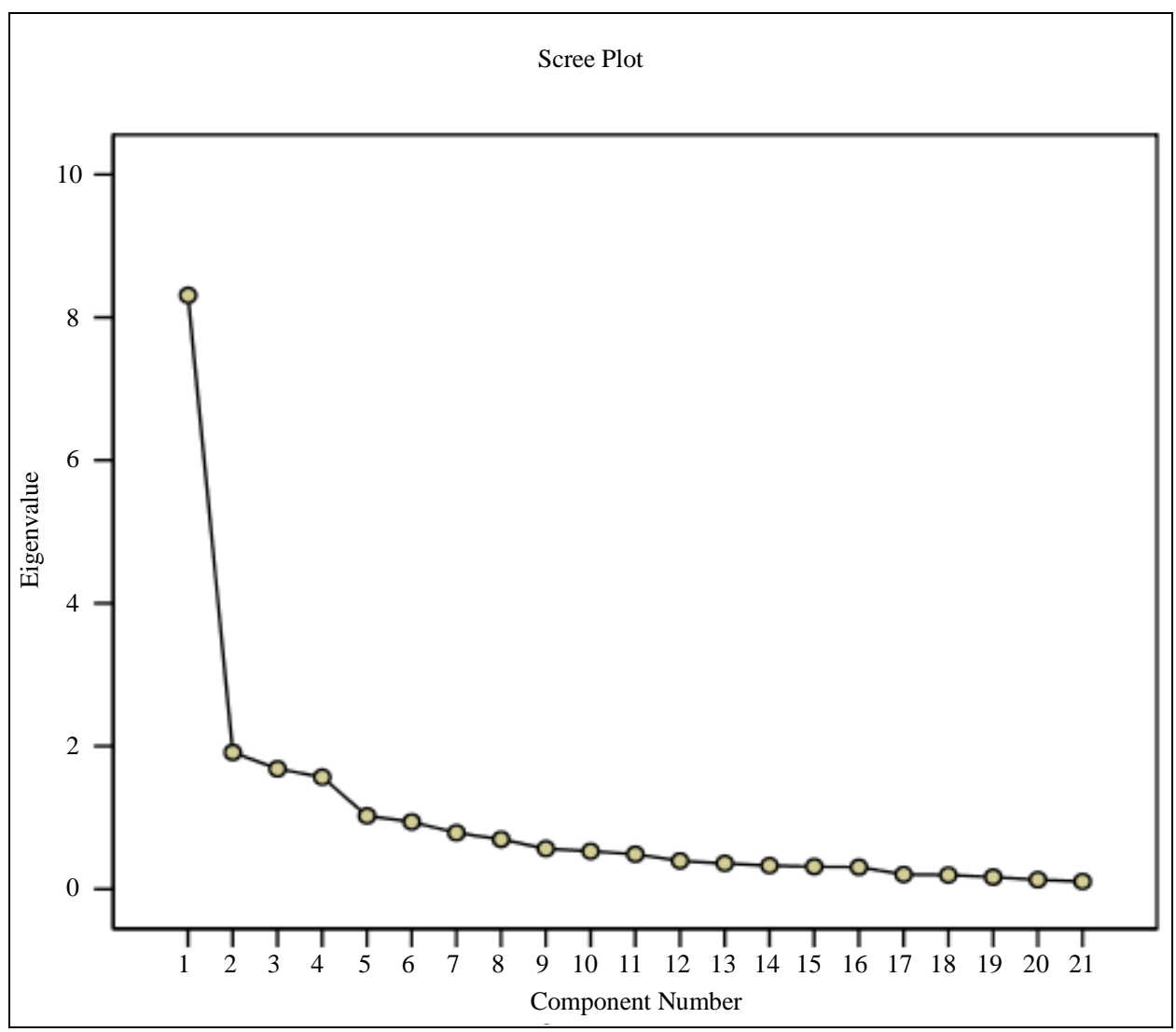

Figure 1. Scree plot of 21 items of family resilience scale. 
Table 3. Exploratory factor analysis on family resilience scale.

\begin{tabular}{|c|c|c|c|c|c|}
\hline & \multicolumn{5}{|c|}{ Factor } \\
\hline & 1 & 2 & 3 & 4 & 5 \\
\hline We make sacrifices for one another & 0.85 & & & & \\
\hline We accommodate one another's needs & 0.85 & & & & \\
\hline Everyone in the family is cooperative & 0.85 & & & & \\
\hline We have good communication within family & 0.82 & & & & \\
\hline We are united & 0.79 & & & & \\
\hline The love we have for one another makes us strong & 0.78 & & & & \\
\hline We do not give up for the sake of one another & 0.74 & & & & \\
\hline We are proactive in dealing with the situation & 0.72 & & & & \\
\hline We are practical in dealing with the situation & 0.65 & & & & \\
\hline We have a pillar in the family and we follow his leadership & 0.52 & & 0.46 & & \\
\hline We redefine the meaning of the situation & & 0.84 & & & \\
\hline We make the situation as normal as possible & & 0.71 & & & \\
\hline We prepare for what might come & 0.30 & 0.68 & & & \\
\hline We belive in adopting a positive outlook & 0.30 & 0.56 & & & \\
\hline We pray to God & & & 0.92 & & \\
\hline We believe in God & & & 0.86 & & \\
\hline We pray to our ancestors for help & & & & 0.94 & \\
\hline We believe that our ancestors will look after us & & & & 0.92 & \\
\hline We control negative emotions & & & & & 0.80 \\
\hline We create a lighter mood in the family & & & 0.31 & & 0.57 \\
\hline We accept the situation & & 0.43 & & & 0.47 \\
\hline
\end{tabular}

Note. Loadings smaller than 0.30 were not shown in the table.

A cutoff at 0.40 was employed for items to be considered loaded under the respective factors. The five factors together accounted for $69 \%$ of the variance. Factor 1 accounted for $40 \%$ of the variance and appeared to consist of items describing how the families come together as units to fight adverse conditions. Factor 2 accounted for $9 \%$, which consisted of items involving attempts to make sense of the situation. Factors 3 and 4 accounted for $8 \%$ and $7 \%$, respectively. Items loaded on these two factors concerned spirituality, with factor 3 focusing on faith in God, while factor 4 described faith in ancestor worship. Factor 5 accounted for $5 \%$ of the variance, and these were items pertaining to efforts to regulate emotions in times of crisis.

Hence, the factors were labeled according to the meaning of the items loaded under them. The five factors are 1) family solidarity, 2) meaning making, 3) spirituality-faith in God, 4) spirituality-ancestor worship, and 5) emotion regulation. However, it is noted that utilizing the criteria of 0.40 , the item "having a pillar in the family" cross-loaded on both factors 1 and 3, while "we accept the situation" cross-loaded on both factors 2 and 5 .

\subsubsection{Factor Analysis (2): Distinct Structure of Family Resilience and Individual Resilience}

Each factor of family resilience and individual resilience was examined for its mean responses, which have been summarized in Table 4.

Results indicate that sharing the same faith in God $(M=4.10)$ and family solidarity $(M=4.03)$ were among the highest-rated sources of family resilience, while the perception of a dynamic and flexible self $(M=3.84)$ was 
the highest-rated source of individual resilience. The correlations among the subfactors of both types of resilience are presented in Table 5.

After the deletion of two multivariate outliers, exploratory factor analysis was performed on the three factors of individual resilience and five factors of family resilience, resulting in two components with eigenvalues greater than one. The scree test (see Figure 2) confirmed the selection of a two-component solution. Hence, two factors were extracted from the principal axis factoring via varimax rotation. The loadings are presented in Table 6.

The five subfactors of family resilience appeared to load under factor 1 , and three subfactors of individual resilience were loaded under factor 2 . Thus, the results indicate two main underlying structures among the eight items: family resilience for factor 1 (accounted for $38 \%$ of the variance) and individual resilience for factor 2 (accounted for $14 \%$ of the variance).

\subsubsection{Mediation Analysis}

Participants' mean responses were examined for individual resilience, family resilience, and subjective wellbeing. Results and their correlations have been summarized in Table 7.

Table 4. Means and standard deviations of the subfactors in family resilience and individual resilience.

\begin{tabular}{cccc}
\hline & Variable & Mean & Standard Deviation \\
\hline Family Resilience & Family solidarity & 4.03 & 0.64 \\
& Meaning making & 3.93 & 0.65 \\
& Spirituality-God & 4.10 & 1.10 \\
& Spirituality-ancestors & 2.33 & 1.16 \\
Endividual Resilience & Emotion regulation & 3.73 & 0.59 \\
& Implicit beliefs of self & 3.84 & 0.54 \\
& Emotional self-regulation & 3.79 & 0.47 \\
& Coping flexibility & 3.74 & 0.50
\end{tabular}

Note: $n=188 .^{* *} p<0.01$

Table 5. Correlations between the subscales of family resilience and individual resilience.

\begin{tabular}{|c|c|c|c|c|c|c|c|c|}
\hline & \multicolumn{8}{|c|}{ Variable } \\
\hline & FS & $\mathrm{MM}$ & SG & SA & ER & IM & ESR & $\mathrm{CP}$ \\
\hline \multicolumn{9}{|l|}{ Family Resilience } \\
\hline \multicolumn{9}{|l|}{ Family solidarity (FS) } \\
\hline Meaning making (MM) & $0.50^{* *}$ & & & & & & & \\
\hline Spirituality-God (SG) & $0.37^{* *}$ & $0.32^{* *}$ & & & & & & \\
\hline Spirituality-ancestors (SA) & $0.24^{* *}$ & 0.14 & 0.02 & & & & & \\
\hline Emotion regulation (ER) & $0.46^{* *}$ & $0.53^{* *}$ & $0.20^{* *}$ & $0.18^{*}$ & & & & \\
\hline \multicolumn{9}{|l|}{ Individual Resilience } \\
\hline Implicit beliefs of self (IM) & $0.16^{*}$ & 0.10 & 0.04 & 0.02 & $0.20^{* *}$ & & & \\
\hline Emotional self-regulation (ESR) & $0.35^{* *}$ & $0.36^{* *}$ & $0.26^{* *}$ & 0.08 & $0.27^{* *}$ & $0.25^{* *}$ & & \\
\hline Coping flexibility & $0.42^{* *}$ & $0.40^{* *}$ & $0.24^{* *}$ & 0.09 & $0.33^{* *}$ & 0.14 & $0.57^{* *}$ & \\
\hline
\end{tabular}

Note: $n=188 .{ }^{* *} p<0.01$; Ind_R = Individual resilience; Fam_R = Family resilience; SWB = Subjective well-being. 


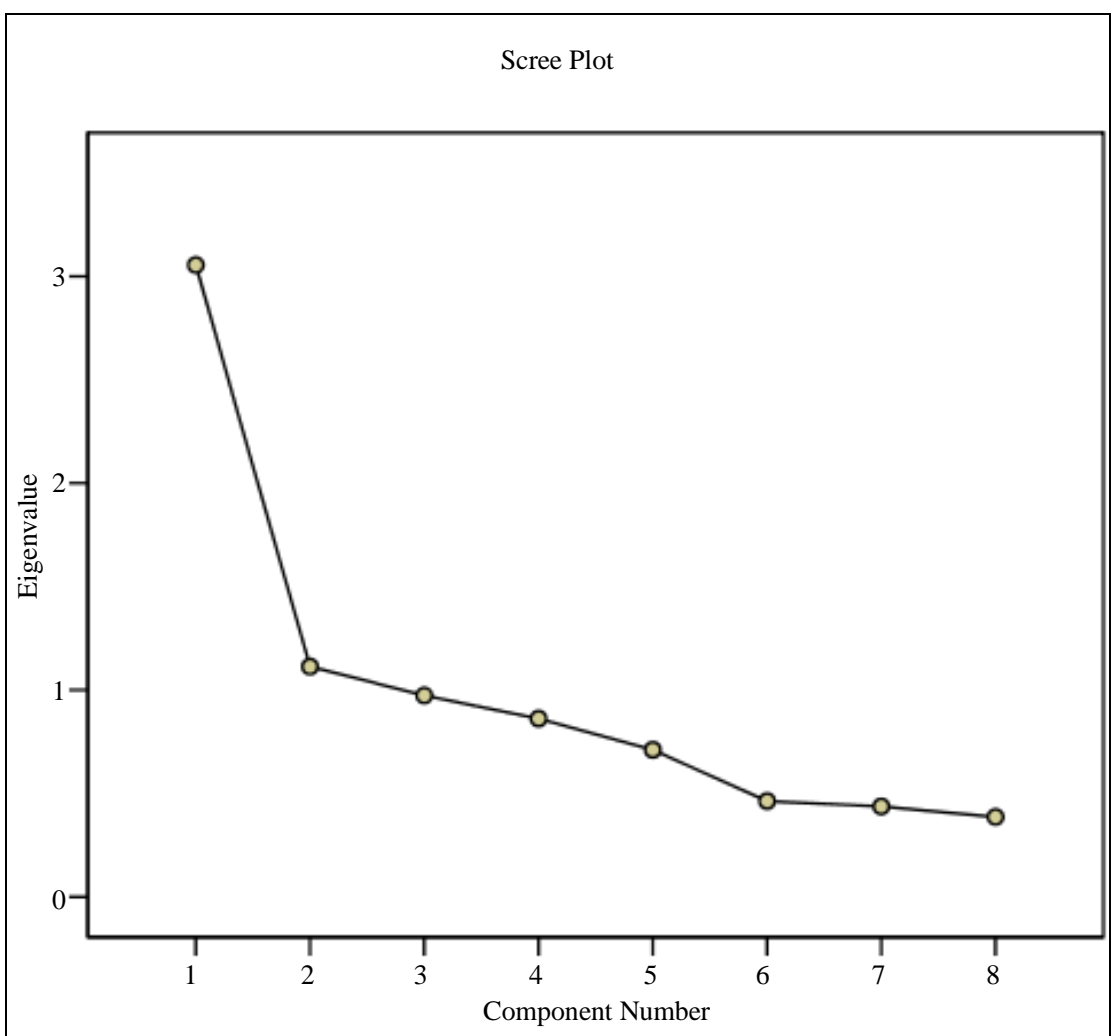

Figure 2. Scree plot of the eight subscales of family resilience and individual resilience.

Table 6. Exploratory factor analysis on the subscales of family resilience and individual resilience.

\begin{tabular}{lcc}
\hline \multicolumn{1}{c}{ Item } & Factor 1 & Factor 2 \\
\hline Family solidarity & 0.70 & 0.32 \\
Meaning making & 0.70 & 0.28 \\
Emotion regulation & 0.64 & 0.20 \\
Spirituality-God & 0.33 & 0.24 \\
Spirituality-ancestors & 0.25 & \\
Emotiional self-regulation & & 0.97 \\
Coping flexibility & 0.34 & 0.55 \\
Implicit beliefs of self & & 0.27 \\
\hline
\end{tabular}

Note: Loadings smaller than .20 were not shown in the table.

Table 7. Means, standard deviations, and correlations between the scales.

\begin{tabular}{|c|c|c|c|c|c|}
\hline & \multicolumn{5}{|c|}{ Variable } \\
\hline & Mean & Standard Deviation & Ind_R & Fam_R & SWB \\
\hline Ind_R & 3.79 & 0.37 & & & \\
\hline Fam_R & 3.81 & 0.51 & $0.46^{* *}$ & & \\
\hline SWB & 3.75 & 0.66 & $0.45^{* *}$ & $0.41^{* *}$ & \\
\hline
\end{tabular}

Note: $\mathrm{n}=188 .{ }^{* *} p<0.01$; Ind_R = Individual resilience; Fam_R = Family resilience; SWB = Subjective well-being. 
Baron \& Kenny (1986) suggest three conditions that need to be fulfilled to claim mediation effects. The conditions required, as applied to the present analysis, were: 1) subjective well-being is regressed on family resilience, 2) individual resilience is regressed on family resilience, and 3) after controlling for family resilience, individual resilience still contributed to the prediction of subjective well-being. Thus, mediation effects would be suggested when there is a substantial reduction of beta for family resilience when the mediator (that is, individual resilience) is present in the regression.

The first two conditions for testing mediation were met, by which subjective well-being was regressed on family resilience $\left(\beta=0.41^{* *}, p<0.01\right)$, and individual resilience was regressed on family resilience $\left(\beta=0.46^{* *}\right.$, $p<0.01$ ). There was a reduction of beta from 0.41 to 0.27 when subjective well-being was regressed on family resilience after accounting for the unique contribution of individual resilience. Hence, the results indicate that individual resilience partially mediated family resilience and subjective well-being (see Table 8).

\subsection{Discussion}

After identifying the family resilience factors, a family resilience scale was constructed as an operationalization of the FR construct. Study 2 tested the internal structure and psychometric properties of the scale and found them to be highly satisfactory. FR positively predicted the subjective well-being and individual resilience measure supporting FR as a measure of family resilience.

Study 2 further tested the relationship between family resilience and individual resilience. Results suggest that family resilience and individual resilience are highly correlated with each other. However, the magnitude of the correlation is lower than 0.50 , indicating that the two factors have less than $25 \%$ of variance with each other, supporting the two factors (that is, family resilience and individual resilience) as related but distinct constructs for individuals.

Owing to the substantial correlation between the two factors, we further tested the potential mediating effects between FR and IR: Results suggested that after partialling out the family resilience factor, the individual resilience factor still contributed to $8 \%$ of the collective subjective well-being for the family. Likewise, when individual resilience was partialled out, family resilience still contributed to $6 \%$ of the variance in the collective family well-being. Therefore, the two variables - resilience at the family level and resilience at the individual level—do not have a spurious relationship: they are independent and different resilience factors.

In addressing the overlapping relationships between the two factors, mediation analysis further suggests that the factors are partially mediated with each other. This can be observed in the shared variance accounted for in the collective family subjective well-being of $24 \%$.

Conceptually, we propose that there may be reciprocal causality between family and individual resilience factors. This indicates that there may be cyclical and reciprocal causality between the two factors. This relationship can be tested in longitudinal developmental studies from the inception of the family and followed through in its developmental processes.

\begin{tabular}{|c|c|c|c|}
\hline & \multicolumn{3}{|c|}{ Subjective Well-being } \\
\hline & $\beta$ & $R^{2}$ & $R^{2}$ change \\
\hline \multicolumn{4}{|l|}{ Equation 1: } \\
\hline Step 1: Fam_R & $0.41^{* *}$ & $0.17^{* *}$ & \\
\hline Step 2: Fam_R + Ind_R & $0.31^{* *}$ & $0.24^{* *}$ & $0.08^{* *}$ \\
\hline \multicolumn{4}{|l|}{ Equation 2: } \\
\hline Step 1: Ind_R & $0.43^{* *}$ & $0.19^{* *}$ & \\
\hline Step 2: Ind_R + Fam_R & $0.27^{* *}$ & $0.24^{* *}$ & $0.06^{* *}$ \\
\hline
\end{tabular}

Note: Fam_R = Family Resilience, Ind_R = Individual Resilience ${ }^{* *} p<0.01$. 


\section{General Discussion}

Our initial observation is that Asian people, when encountering great adversity, often cope not as individuals, but rather work together with their closest collective group: the family. This informal observation was confirmed in our first study where we interviewed nurses who were quarantined after they were assigned to work with SARS patients. The participants reported that they drew both physical resources and psychological support from their families. This confirms the literature on family studies stating that in times of crisis, the family, as a functioning unit, also goes through the stress-and-coping process (Bentelspacher et al., 1994; McCubbin \& Patterson, 1982; Patterson, 1998, 2002). However, some families fare better than others do. We searched for resilience factors that could help the individual and the family weather the challenge better. Our search with a series of qualitative studies in Study 1 identified a set of family resilience factors: family solidarity, meaning making, spirituality—God, spirituality—ancestors, and emotional regulation.

Comparing these factors with the family resilience factors identified in Western literature (for instance, Black \& Lobo, 2008; Cohen et al., 2002; Walsh, 1998), at the structural level, the emphasis on adherence to roles and responsibilities and willingness to sacrifice for and support the family seems to differentiate these Asian families from North American families. These results are consistent with commonly reported observations of Asian cultures, where people place greater emphasis on the collective than on the individual and, within the family, the emphasis on roles and structural stability is as important as the affective bond between family members.

Study 2 tested the relationship between family resilience and individual resilience. It was found that family resilience is not a spurious construct related to individual resilience (Chang, 2007; Chang \& Lim, 2007; Rutter, 1987, 1999). The two factors significantly overlap but are distinct from each other, each contributing independently and significantly to the outcome variable of the collective subjective well-being.

The relationship between the two variables is unclear at present, however. Mediation analysis suggests that they partially mediate each other's effects on the outcome. We hypothesize that there may be cyclical and mutual causality between the family resilience factor and the individual resilience factor. Yet this hypothesis can be tested only in longitudinal studies. Our cross-sectional studies can only provide a pilot effort in understanding the relationship between the two factors.

\section{General Conclusion and Discussion}

The two studies presented in this report identified a set of factors that constitute the family resilience factors, based on which a family resilience (FR) scale was developed. We found that FR factors contributed significantly to the adaptive outcome (that is, subjective well-being) and that FR was highly correlated with psychological resilience at the individual level (that is, individual resilience). However, further analysis found that they were separate factors, and each made a significant contribution to individual and collective family welfare.

We also found that the FR identified in the present study differs from the known North American FR (Walsh, 1998) in its emphasis on structural/role stability rather than on flexibility, as well as its emphasis on individuals' altruism for the family. These differences are consistent with our understanding of the differences in values between the East and the West (Hofstede, 1980). Furthermore, the higher emphasis on role structural stability is consistent with our own earlier observation of Singaporeans-a modern Asian community that places great emphasis on social institutions as well as individual competence (Chang, Wong, \& Koh, 2003).

More importantly, results from current studies confirm a common observation in Asia: in times of crisis, Asians react and manage the challenges collectively, reacting as a unit rather than as separate individuals. This conclusion has been confirmed in a series of recent disasters in Asia: following tsunamis, earthquakes, and volcanic eruptions, rescue teams found strong social support and solidarity at the family and community levels.

The finding that the family reacts and copes together in times of major disasters affirms an age-old observation of the Asian populations, even in modern and affluent Asian communities such as Singapore. The identification of family resilience factors and the development of the family resilience scale can have a wide range of application in education, health, and mental health policies and practice.

Ethnic differences between the groups were apparent when Malay families coped by involving the family in routine religious practices, while Chinese families coped by looking up to the pillar in the family to lead them in times of crisis. Chinese families also uniquely exhibited a strong inclination toward control and suppression of negative emotions, which contrasted with Malay families’ relatively proactive attempts to create a lighter mood in the family. However, these minor ethnic differences did not overshadow the overwhelming similarity in FR 
across the two Singaporean ethnic groups. With the cross-sectional design, we cannot ascertain the causal direction between family and individual resilience, but the close correlation suggests mutual reciprocal causality that awaits further validation.

With this study, we further propose that for Asian populations, especially in research on individual psychological and physical health, it is important to pay attention to the family of the individual participants, for individuals often do not function alone but as integrated members of families or immediate collectives. Future research should test the efficacy of FR in different domains of life's challenges: major health problems, critical examinations, and migration, for instance.

\section{Limitations}

A number of limitations were found in the current study. The study was conducted with a cross-sectional design to take advantage of a mass health crisis that threatened a large portion of the population to understand the origin and development of family resilience concerning individual psychological resilience. The second limitation concerns the type of family involved in the present study, which concerned mainly spousal relationships. Although we think the family factors apply to the entire family and major types of families (as long as the family functions as a unit of operation), there may be variations in the different relationships within the family, for instance, spousal versus parent-child or parent-child versus siblings. Further, the individual participants who directly experienced life challenges were nurses, who were in a caring profession and mostly female; they may be naturally more inclined to be altruistic toward their families. We may need to work with more male participants and with different types of life challenges to generalize the results to these populations. We propose that the FR construct and the FR-inspired instrument be validated further with other Asian populations and across genders and ages to test its generalizability and range of application.

\section{References}

Baron, R. M., \& Kelley, D. A. (1986). The Moderator-Mediator Variable Distinction in Social Psychological Research: Conceptual, Strategic, and Statistical Considerations. Journal of Personality and Social Psychology, 5, 1173-1182. http://dx.doi.org/10.1037/0022-3514.51.6.1173

Bentelspacher, C. E., Chitran, S., \& Rahman, M. (1994). Coping and Adaptation Patterns among Chinese, Indian, and Malay Families Caring for a Mentally Ill Relative. Families in Society, 75, 287-300.

Black, K., \& Lobo, M. (2008). The Conceptual Review of Family Resilience Factors. Journal of Family Nursing, $14,33-65$. http://dx.doi.org/10.1177/1074840707312237

Bonanno, G. A. (2004). Have We Underestimated the Human Capacity to Thrive After Extremely Aversive Events? American Psychologist, 59, 20-28. http://dx.doi.org/10.1037/0003-066X.59.1.20

Bonanno, G. A., Ho, S., Chan, J. C. K., Kwong, R. S. Y., Cheung, C. K. Y., \& Wong, C. P. (2008). Psychological Resilience and Dysfunction among Hospital Survivors of the SARS Epidemic in Hong Kong: A Latent Class Approach. Journal of Health Psychology, 27, 659-667. http://dx.doi.org/10.1037/0278-6133.27.5.659

Bronfenbrenner, U. (1986). Ecology of Family as a Context for Human Development: Research Perspectives. Development of Psychopathology, 22, 723-742. http://dx.doi.org/10.1037/0012-1649.22.6.723

Carver, C. S. (1998). Resilience and Thriving: Issues and Models and Linkages. Journal of Social Issues, 54, 245-266. http://dx.doi.org/10.1111/j.1540-4560.1998.tb01217.x

Chang, W. C. (2007). Forged in Fire: Indigenous Resilience Factors of Singaporean Chinese and Malays. Advances in Asian Social Psychology, 4, 17-39.

Chang, W. C., \& Lim, L. H. (2007). Resilience and Subjective Wellbeing in an Asian Context: Singapore. Journal of Psychology in the Chinese Societies, 8, 47-69.

Chang, W. C., Wong, W. K., \& Koh, J. B. K. (2003). Chinese Values in Singapore: Traditional and Modern. Asian Journal of Social Psychology, 6, 5-29. http://dx.doi.org/10.1111/1467-839X.t01-1-00007

Chu, R. (2002). Asian Subjective Wellbeing. Master's Thesis, National University of Psychology.

Cohen, O., Slonim, I., Finzi, R., \& Leichtentritt, R. D. (2002). Family Resilience: Israeli Mothers’ Perspectives. The American Journal of Family Therapy, 30, 173-187. http://dx.doi.org/10.1080/019261802753573876

DeHaan, L., Hawley, D. R., \& Deal, J. E. (2002). Operationalizing Family Resilience: A Methodological Strategy. The American Journal of Family Therapy, 30, 275-291. http://dx.doi.org/10.1080/01926180290033439

Fletcher, D., \& Sarkar, M. (2013). Psychological Resilience: A Review and Critique of Definitions, Concepts and Theory. 
European Psychologist, 18, 12-23. http://dx.doi.org/10.1027/1016-9040/a000124

Garmezy, N. (1985). Stress-Resistant Children: The Search for Protective Factors. In J. E. Stevenson (Ed.), Recent Research in Developmental Psychopathology: Journal of Child Psychology and Psychiatry Book Supplement, No. 4 (pp. 213-233). Oxford: Pergamon.

Hofstede, G. (1980). Culture’s Consequences. Beverly Hills, CA: Sage publications.

Lazarus, R., \& Folkman, S. (1984). Stress, Appraisal and Coping. New York: Springer.

Lee, I., Lee, E., Kim, H. S., Park, Y. S., Song, M., \& Park, Y. H. (2004). Concept Development of Family Resilience: A Study of Korean Families with a Chronically Ill Child. Journal of Clinical Nursing, 13, 636-645. http://dx.doi.org/10.1111/j.1365-2702.2004.00845.x

Luthar, S. S. (2006). Resilience in Development: A Synthesis of Research across Five Decades. In D. Cicchetti, \& D. J. Cohen (Eds.), Developmental Psychopathology: Risk Disorder and Adaptation (pp. 739-795). New York: Wiley.

Marsten, A. S., \& Obradovic, J. (2008). Disaster Preparation and Recovery: Lessons from Research on Resilience in Human Development. Ecology and Society, 13, 9.

McCubbin, H., \& Patterson, J. (1982). Family Adaptation to Crises. In H. McCubbin, A. Cauble, \& J. Patterson (Eds.), Family Stress, Coping and Social Support (pp. 26-47). Springfield, IL: Thomas.

Patterson, J. M. (1988). Families Experiencing Stress: The Family Adjustment and Adaptation Response Model. Family System Medicine, 5, 202-237. http://dx.doi.org/10.1037/h0089739

Patterson, J. M. (2002). Understanding Family Resilience. Journal of Clinical Psychology, 58, 233-246. http://dx.doi.org/10.1002/jclp.10019

Rutter, M. (1987). Psychosocial Resilience and Protective Mechanisms. American Journal of Orthopsychiatry, 57, $316-331$. http://dx.doi.org/10.1111/j.1939-0025.1987.tb03541.x

Rutter, M. (1999). Resilience Concepts and Findings: Implications for Family Therapy. Journal of Family Therapy, 2, 119144. http://dx.doi.org/10.1111/1467-6427.00108

Seligman, M. E. P., \& Csikszentmihalyi, M. (2000). Positive Psychology: An Introduction. American Psychologist, 55, 5-14. http://dx.doi.org/10.1037/0003-066X.55.1.5

Stinnett, N., \& De Frain, J. (1985). Secrets of Strong Families. Boston, MA: Little Brown.

Walsh, F. (1998a). Strengthening Family Resilience. New York: Guildford Press.

Walsh, F. (1998b). Beliefs, Spirituality and Transcendence: Key to Family Resilience. In F. Walsh, \& Goldrick, M. (Eds.), Revisioning Family Therapy: Race, Culture, and Gender in Clinical Practice (pp. 62-77). New York: Guildford Press.

Walsh, F. (2003). Family Resilience: A Framework for Clinical Practice. Family Process, 42, 1-18. http://dx.doi.org/10.1111/j.1545-5300.2003.00001.x 